\title{
BMJ Open Rationale and protocol for using a smartphone application to study autism spectrum disorders: SMARTAUTISM
}

\author{
Olivier Bonnot, ${ }^{1}$ Dominique Bonneau, ${ }^{2}$ Aude Doudard, ${ }^{3,4}$ Philippe Duverger ${ }^{3,4}$
}

To cite: Bonnot 0 ,

Bonneau D, Doudard A, et al. Rationale and protocol for using a smartphone application to study autism spectrum disorders: SMARTAUTISM. BMJ Open 2016;6:e012135. doi:10.1136/bmjopen-2016012135

- Prepublication history for this paper is available online. To view these files please visit the journal online (http://dx.doi.org/10.1136/ bmjopen-2016-012135).

Received 5 April 2016 Revised 1 September 2016 Accepted 20 October 2016

\section{CrossMark}

\footnotetext{
${ }^{1}$ Child and Adolescent Psychiatry Department, University Hospital of Nantes, Nantes, France

${ }^{2}$ Department of Biochemistry and Genetics, $\mathrm{CHU}$ Angers, Angers, France

${ }^{3}$ Resource Center for Autism (CRA) Loire Valley, CHU Angers, Angers, France ${ }^{4}$ Psychiatry and Adolescent Department, CHU Angers, Angers, France
}

Correspondence to Professor Olivier Bonnot; olivier.bonnot@gmail.com

\section{ABSTRACT}

Introduction: Longitudinal studies on the evolution of autism spectrum disorder (ASD) symptoms are limited and have primarily used repeated measurements performed several months apart. However, measurements of changes in everyday life should more closely reflect the 'real life' of the patient and his or her family. We propose to study the child's ASD symptoms and their effect on the quality of life, psychological status and anxiety of the child's parents over a 6-month period using SMARTAUTISM, a smartphone application.

Method and analysis: This is a prospective, longitudinal, exploratory, open study with a 6-month follow-up period. Data will be recorded longitudinally over multiple weeks under natural conditions. The factors affecting the quality of life and anxiety of parents of children with ASD and the children's functional symptoms will be examined, and the feasibility of using a smartphone application designed for parents of ASD patients will be assessed. Primary objective: Explore the evolution of a child's behaviour over 6 months and the (psychological and social) effects of these changes on the family. Secondary objective: Assess the feasibility of our application by examining the filling rate and application usage by parents for 6 months. 100 families containing 1 child diagnosed with ASD will be included. At baseline, sociodemographic, psychiatric and medical data will be recorded. The correlations of the general epidemiological variables (primary outcome measure) will be evaluated via multivariate analysis. The application filling rate (relative to the ideal filling rate) will be used to assess the feasibility of the application (secondary outcome measure).

Ethics and dissemination: The SMARTAUTISM study has the approval of the local ethics committee, and data security will be ensured via the use of encryption and a secure medical server. The use of this application will be proposed at autism resource centres across France.

\section{CONTEXT}

Information communication technologies, including online or offline smartphone applications, are a currently rapidly progressing

\section{Strengths and limitations of this study}

- First smartphone application enabling ecological momentary assessment of behavioural symptoms of children with autism spectrum disorder and quality of life of parents.

- Six months of follow-up data.

- Application providing easy access and readable feedback to enhance parents' motivation in the filling process.

- Subjective evaluation is provided by parents.

- Items are simple, and unexpected situations may not be recordable.

reality in medicine. There are $\sim 100000$ apps in the Android or Apple market. ${ }^{1}$ Many of these apps, including coaching, well-being advice or sports applications, have no medical purpose but are labelled as 'health' applications. However, in the field of psychiatry, as for all medical specialties, apps are more than just a trend. In fact, apps may represent a revolution in research and doctorpatient relationships.

Apps may be used in different ways. The simplest way to use this device-based technology is to provide access to healthcare through smartphone applications in countries where the availability of equipment and medical personnel is limited. ${ }^{2}$ Further, this technology can be conceptualised as mainstream news media to reduce stigmatisation. ${ }^{2}$ Apps may also be helpful in remote support via SMS, voice or visual phone consultations for medical purpose.

More importantly, medicine, as well as psychiatry, requires data from patients or their families. These data might be obtained from questionnaires that can be completed under natural conditions; this technique is referred to as ecological momentary assessment $\left(\mathrm{EMA}^{3}\right)$ or the experience sampling method. ${ }^{4}$ The first EMA experiments used beepers to remind users to complete the questionnaires on a computer; in general, 
the results for compliance and quality were disappointing, and the filling schedules were not respected. ${ }^{5}$ Since the release of PDAs and smartphones, individuals are carrying their device everywhere. This is a breakthrough for medical research in various fields, but psychological status and behaviour, including behavioural addictions, ${ }^{6}$ eating behaviors, ${ }^{7}$ anxiety ${ }^{8}$ and severe psychiatric disorders, such as bipolar disorder ${ }^{9}$ or schizophrenia, appear to be particularly appropriate topics for applicationbased studies. ${ }^{10}$ Apps are very useful for evaluating the social functioning, quality of life and psychological status of individuals. As demonstrated in anxiety disorder, EMA using smartphones is a practical and effective approach, and the data generated have higher quality than data generated via other methods. ${ }^{8}$

There are two major issues regarding the use of apps in psychiatry. The first issue is their feasibility (Will participants use the app?), and the second is their efficiency.

The feasibility of an application-based method is important to assess. One of the few studies measuring this parameter suggested that the filling rate varies depending on the type and duration of app use (for filling and educational purposes) but might be as high as $70 \% .{ }^{11}$ The literature in this domain is limited and largely consists of preliminary data or study protocols with varying durations (days to weeks) or performed on a small number of participants (10-100 participants). Interestingly, a recent survey showed that $77 \%$ of a US population of patients with a psychiatric disorder owned a smartphone and that these patients would be willing to use their smartphone to register their mental state. ${ }^{12}$

The core concept underlying EMA is the collection of behavioural data provided by the participant (and potentially the participant's family members or caregivers). These naturalistic data are a novelty and will affect our understanding of disorders as well as therapeutic relationships (see examples for eating disorders $^{67}{ }^{13}$ or addictions ${ }^{14}$ ). However, the efficacy of EMA depends on the patient's desire to provide data that are useful collectively but not individually. Few research programmes include a specific level of feedback by the application (see rare examples for schizophrenia ${ }^{13}$ or bipolar disorder ${ }^{915} 16$ ).

App-generated feedback might be an incentive for filling the application, but it also represents a potentially important change in the doctor-patient relationship. One study of particular interest includes 52 participants and proposes to compare behavioural interpersonal therapy with therapy available on demand using a smartphone or tablet (dematerialised adaptation) over a 5 -month period. ${ }^{17}$ The percentage of treatment responders was $54 \%$ for the 'mobile' group compared with $8 \%$ for the conventional group, which is surprisingly low. Among other factors, a certain degree of feedback appears to increase acceptance and filling rates; for example, in a sample of patients with schizophrenia, one recent work showed an acceptable level of use $(50 \%$ spontaneous use and 38\% application-induced use) and excellent acceptance (90\% satisfied) for an application based on psycho-educational support (information, resources, etc) with a small portion of self-care and feedback. ${ }^{18}$

In summary, combining EMA with feedback is a promising approach for (1) increasing the feasibility of smartphone app-based research methods and (2) promoting a qualitative change in the doctor-patient relationship. We developed such an application in the autism spectrum disorder (ASD) field.

\section{The SMARTAUTISM project}

This ongoing (starting 1 September 2016) study received funding from the Regional Health Agency (ARS) and the Autism Resource Centre (CRA) of the Loire Valley, as well as a grant from the National Center for Autonomy (CNSA)/Research Institute of Epidemiology and Public Health (IRESP).

ASD symptoms are now better understood, but ASD studies are most often transversal or mostly retrospective. Actual longitudinal studies on symptom evolution remain limited, and researchers continue to use repeated measurements recorded several months apart. ${ }^{15} \quad 1920$ However, daily changes should be more accurate and, above all, should more closely reflect the 'real life' of the patient and his or her family.

Children with ASD and their parents often face difficult situations daily that are related to behavioural symptoms (agitation, feeding difficulties, sleep disturbances, obsessive behaviour, refusal of authority, etc). Further, the parents' quality of life is strongly affected by everyday life with their children with ASD. ${ }^{21}{ }^{22}$ Data on these behavioural disorders are currently being collected in a transverse manner using surveys, questionnaires and parental recall. This mode of data collection causes a clear, and classical, bias in all studies.

Our goal is to study a child's ASD symptoms and their impact on a family's quality of life, psychological status and anxiety over a long time period (6 months) using a daily information source that includes parental observations. Moreover, our EMA application that includes feedback could be useful for facilitating parents' necessary adaptive educational behaviour and for informing the doctor (who will have access to the data during regular consultations).

In addition to quality of life, anxiety of the parents is a dimension that we will consider. Parents of children with ASD have greater anxiety levels than parents of children without a developmental disorders (for a recent meta-analysis on this participant, see, ${ }^{23}$ which included 15 studies). The major autism symptoms, communication disorders and aggression, are the most important stressors. ${ }^{24}$ Most of these reports describe cross-sectional studies that evaluated parents using a single semistructured interview or questionnaire. 
An EMA app has never been used to study the parents of children with ASD in terms of quality of life and anxiety level. Additionally, there are no epidemiological studies of this kind in the literature.

The SMARTAUTISM application represents a combination of a classic EMA with feedback that may be used by parents to adapt their educational behaviour and used by doctors to better understand their patients and propose more accurate treatments. The parents must provide regular ratings (twice a week at least) for a long period (6 months for our study). In our proposal, the parental rating time and application design are two main factors.

1. The rating time will range from 3 to $10 \mathrm{~min}$, depending on the responses provided. The questions will be simple and user-oriented (figures 1 and 2). We will ask questions about sleep; behaviour; ASD symptoms, such as stereotyping or withdrawal; and social life, such as lunch period or leisure activities. With respect to the parents, the questions will be about anxiety and psychological state of mind in addition to quality-of-life elements (ability to function in everyday domestic/professional/friendly tasks and social contact). To save time, we will use a visual analogue scale (VAS). The VAS is an accurate tool that facilitates repetitive measurements in psychiatry (eg, see the MATHYS experiment ${ }^{25}$ ). Users will provide a rating from 0 to 10 by scrolling their finger on the touch screen (figure 3). To maintain a short filling time, all responses will be selected from a list of words yes/no buttons or from a VAS. Our choice is radical, and we anticipate that the relatively reduced quality of the data will be counterbalanced by the volume of data.

2. The design of the application was a continuous concern throughout the development process; we have research collaborations with a specialised private company with expertise on this topic. Each screen provides information using soft colour and typography. SMARTAUTISM is also personalised. For example, users may use a particular child's picture as the wallpaper (figure 4).

SMARTAUTISM provides a menu section with general information about ASD and local resources (healthcare facilities, emergency numbers, etc). It also allows the user to change the settings, particularly the rating reminder notification. The application also provides two summary screens for the data collected from the first input (a feedback). These screens are user-friendly and readable. Users will be able to have a graphic view (current and past) of their child's behaviour after each assessment. This visual feedback will be presented to the user and can be shown to a third party (such as a doctor).

We propose a study (1) to evaluate the feasibility of using EMA to investigate a child's ASD symptoms as well

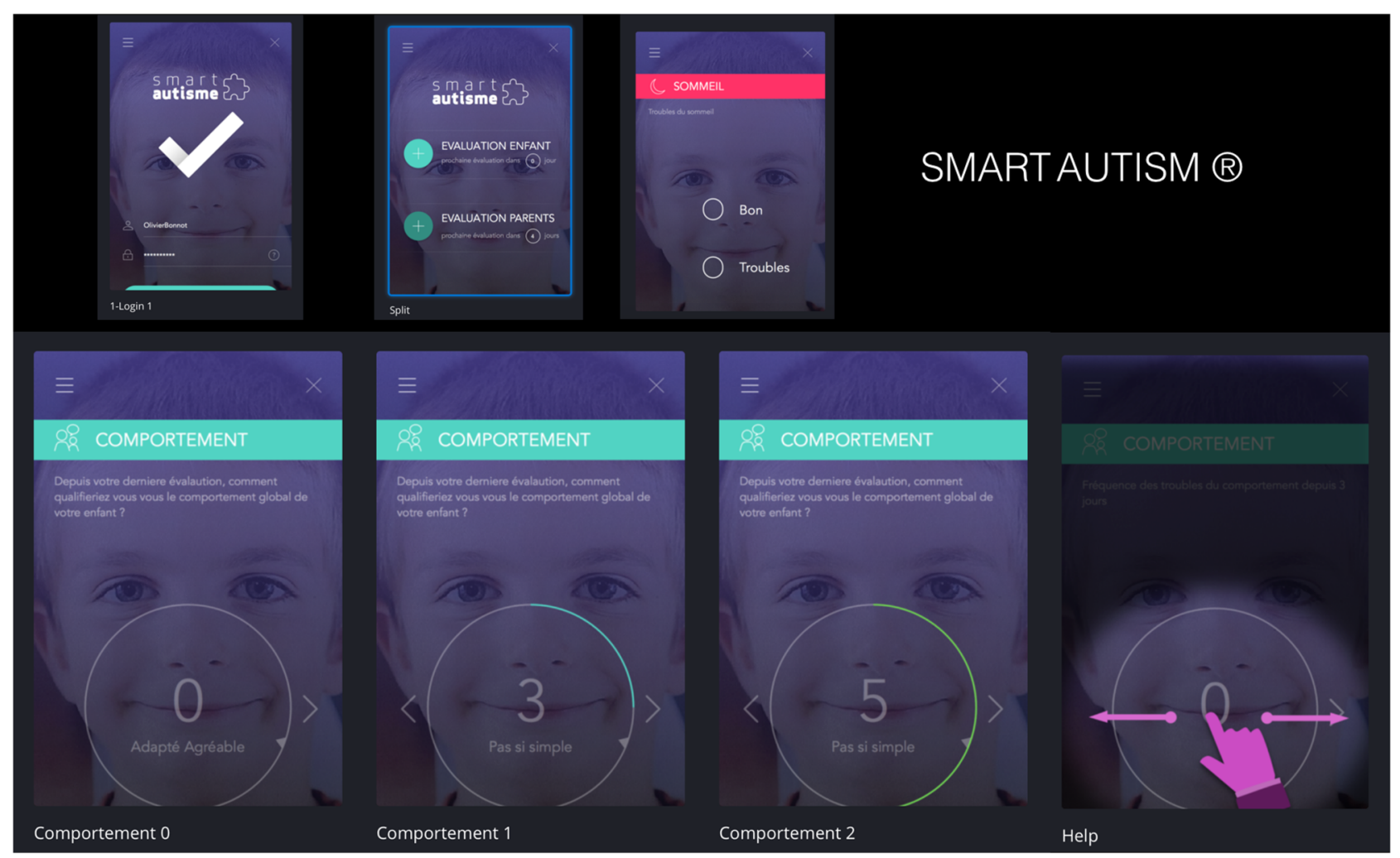

Figure 1 Snapshots from the SMARTAUTISM application. 


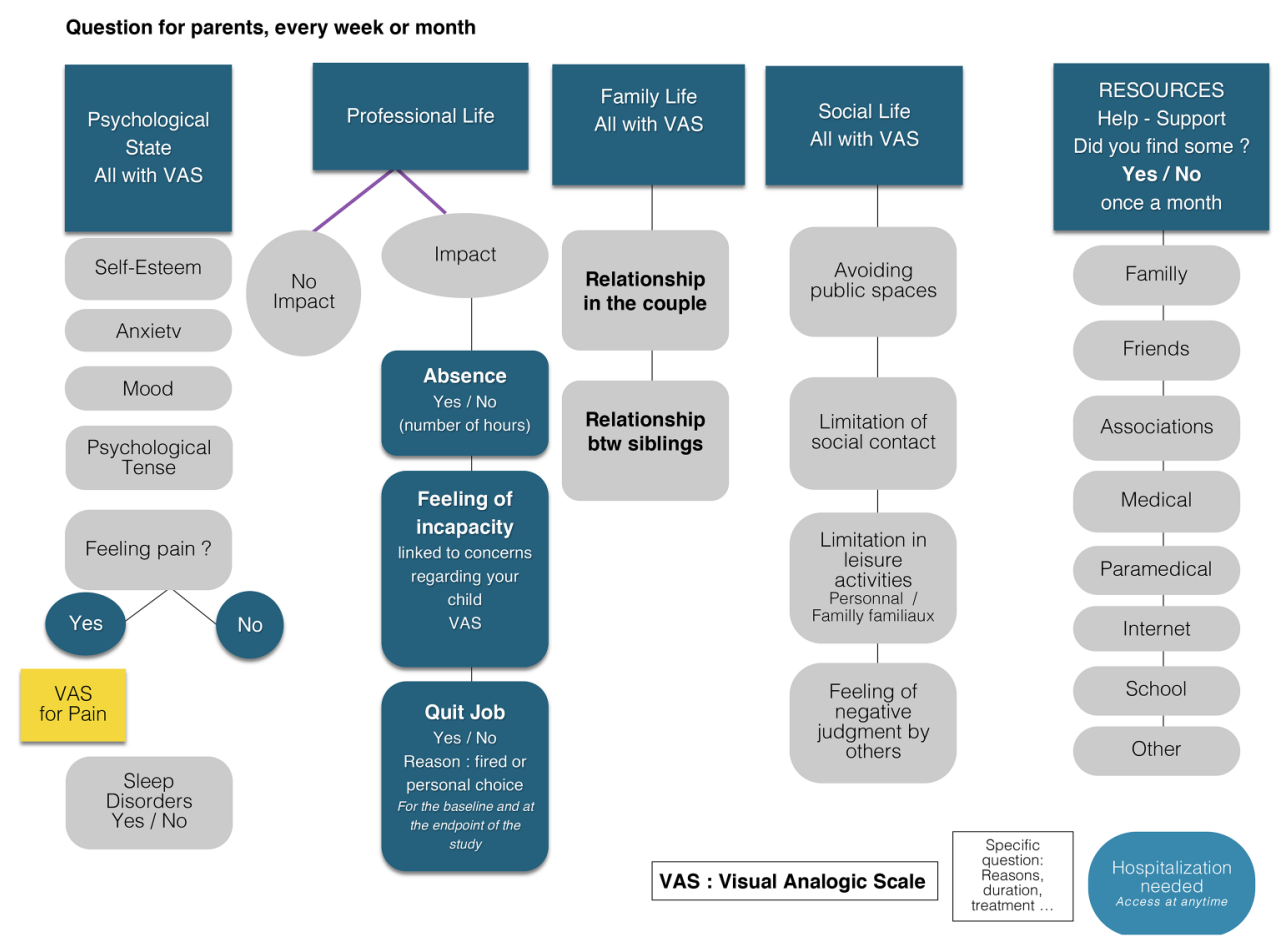

Figure 2 Questions regarding psychology and quality of life for parents.

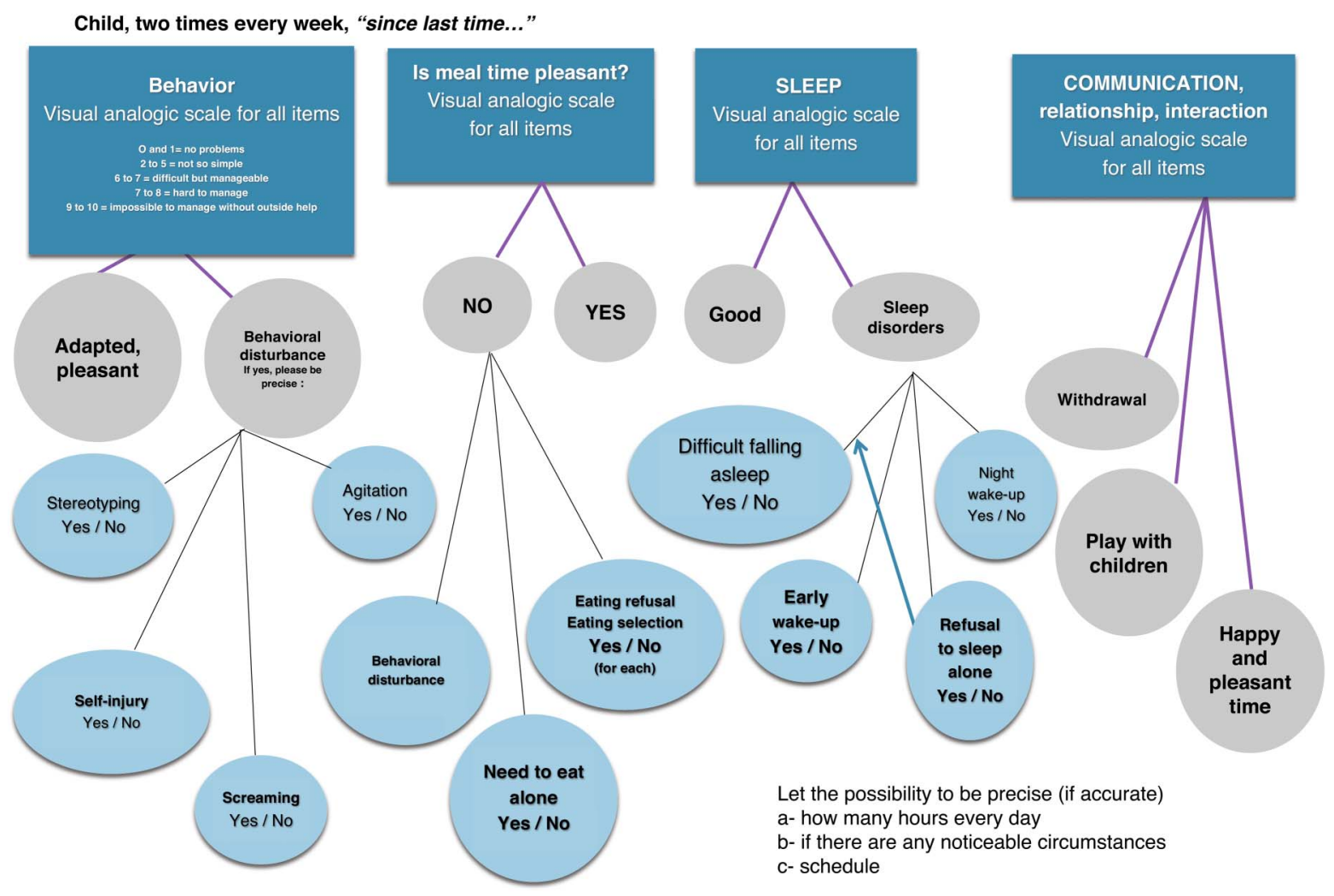

Figure 3 Questions regarding behaviour of individuals with autism spectrum disorders.

as a parent's quality of life, psychological state and daily anxiety level over 6 months and (2) to collect epidemiological data. The SMARTAUTISM study will begin in September 2016.

\section{OBJECTIVES AND ASSESSMENT CRITERIA Primary objective}

The primary main objective is to assess the feasibility of this innovative app-based approach. 


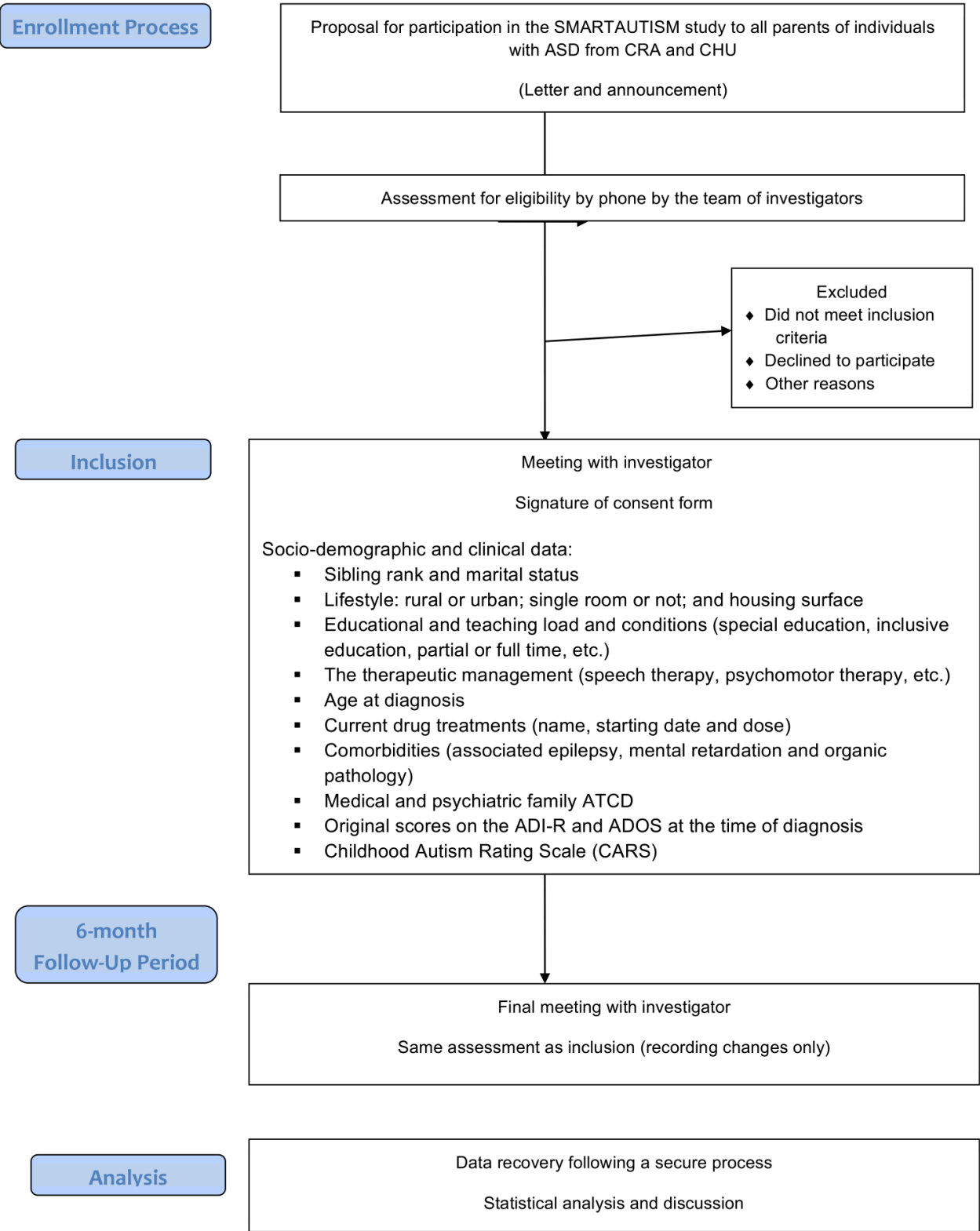

Figure 4 SMARTAUTISM protocol diagram flow.

\section{Primary criteria}

The primary criteria will be the filling rate $(\mathrm{R})$ and the use of our application by the parents over 6 months.

$$
\frac{\text { Number of fillings }}{60^{*}}=\mathrm{R}
$$

where $*$ indicates every 3 days over 6 months $=6 \times 30 /$ $3=60$ filling dates.

\section{Secondary objectives}

Our secondary objective is an epidemiological study on the evolution of a child's behaviour over 6 months and the simultaneous self-reported psychological status of the family.

\section{Secondary criteria}

Secondary criteria include data from the application, which is filled by the parents, predominantly using a VAS from 0 (no) to 10 (severe) to measure the following variables:

- For the child (see figure 3): (1) behaviour with pairs and communication, (2) sleep disorders, (3) eating disorders and (4) general behaviour.

- For parents: (see figure 2): (1) psychological status (self-esteem, anxiety, sleep, mood, pain and psychological stress), (2) professional life, (3) personal life, (4) social life and (5) support and resources.

We will also be able to access more descriptive data regarding the use of the application, such as (1) the duration of use, (2) the time of use and (3) the rate of complete filling. 


\section{Methodology}

This is a prospective, longitudinal, exploratory, open study with a 6-month follow-up period to assess the feasibility of using a smartphone application designed for parents of patients with ASD and to longitudinally collect data on the quality of life and anxiety of parents of children with ASD and the children's functional symptoms over multiple weeks under natural conditions.

\section{Population}

We propose to study individuals diagnosed with ASD based on National Health Authority (HAS) recommendations (on ADI-R diagnoses) from 3 to 16 years of age in the Pays de la Loire region. We propose to include 100 families. The study is exploratory in nature; thus, randomising patients is unnecessary. We will include the first 100 families agreeing to participate in the study and meeting the inclusion criteria. All participants will be recruited from the Department of Child and Adolescent Psychiatry of CHU de Nantes and from the Regional Center for Autism (CRA des Pays de la Loire).

Inclusion criteria:

- Having a child with an ASD diagnosis based on the ICD 10 criteria.

- Having at least one smartphone (iOS Apple or Android).

- Signing the consent form for participation.

Exclusion criteria:

- Having several children with an ASD diagnosis.

- Having children living in more than two areas.

\section{Method}

At the inclusion visit, parents will receive a code to download the app from the 'application store' of their choice (depending on their smartphone) and sign an informed consent form (see flow chart, figure 4).

All type 1 data from the inclusion visit will be stored in an electronic database and in the smartphone application (during the setup process).

This initial evaluation by the investigator will include the following type 1 data:

- Sibling rank and marital status,

- Lifestyle: rural or urban; single or multiple occupants of the child's bedroom and housing type and surface,

- Educational and teaching load and conditions (special education, inclusive education, partial or full time, etc), and

- The therapeutic management (speech therapy, psychomotor therapy, etc).

Type 2 data will be stored in the electronic database only:

- Age at diagnosis,

- Current drug treatments (name, starting date and dose),

- Comorbidities (associated epilepsy, mental retardation and organic pathology),

- Medical and psychiatric family ATCD,
- Original scores on the ADI-R and ADOS at the time of diagnosis, and

- Scores on the French version of the Childhood Autism Rating Scale ${ }^{26}$ (CARS) (http://www.autisme. qc.ca) at baseline and at the end of the study.

A default schedule for filling out the routine application questions (as shown in figures 2 and 3) with notification reminders will then be proposed to the parents. The default settings can be modified by the parents if necessary but must include at least three fillings each week. Missing data will be included in the assessments. Each filling will require a short time period $(<10 \mathrm{~min}$ in the longest configuration, if all answers are yes). The key questions are closed-ended (yes/no), and a VAS is primarily used to evaluate the degrees of statuses, symptoms and disorders.

The first question concerns the environmental context in the days surrounding the filling date (unusual, holiday, etc). With respect to the children, the questions will focus on sleep, food and behaviour concerning themselves and others (such as aggressiveness, agitation, stereotypic behaviours or cries). To avoid redundant or inappropriate questions, the questions will be presented as an algorithm (see figures 1 and 2).

In cases in which both parents have a smartphone, both will have the application, and filling may be performed by either parent. The data will be aggregated per family. Simultaneous assessments by both parents are unnecessary in this study.

During the 6-month inclusion period and the 6-month follow-up period, we will record encrypted data from the smartphone when the parents are connected to a Wi-Fi network using a secure medical server (16-digit encryption key+a personal temporary code for access to data).

We have developed a qualitative questionnaire for the participants and the patients' doctors as well as a semidirected, face-to-face interview to assess the change in the doctor-patient relationship after using the application.

\section{Statistical analysis}

For this feasibility study, the primary end point (which corresponds to the primary objective) will be the application filling rate, which is calculated relative to the ideal filling rate (3 days per week every week) and considers the number of fillings (of at least half of the items requested).

The secondary end points will require more precise analyses, such as examination of the variation in the rate of responding to at least half of the requested items over time. We will collect a considerable amount of data due to the number of variables; as such, we will be able to perform exploratory epidemiology on this essential and unique database using descriptive and analytical statistics.

Generally, we will compare two groups: the group with a single place of family life and the group with two 
places of family life. The data encoded by the parents are subjective, but we do not distinguish the data on a child according to the parent. However, the quality of life data will be distinguished based on the parent who provided the rating. Thus, we will include 100 patients and more than 100 parents reporting on quality of life.

The analysis will be performed in two stages: (1) a longitudinal data analysis of each group (parents and children) and (2) multivariate analyses on the relationships between variables specific to the parents and variables specific to the children.

\section{Safety issues}

Protection of personal data is a very important issue with very restrictive regulations in France and Europe. Therefore, to obtain approval from the National Center for Informatics and Liberty (CNIL, http://www.cnil.fr), we have designed a secure pathway of data handling. Data will be stored in the application on the parents' smartphones and will be inaccessible by unauthorised people (an individual code will be required on application opening). In particular, there is no internet connection needed to run the application. As part of the research, and after considering the opinion of the CNIL, families included in this study will transmit these data to the investigators using a strict, secure, five-step process:

1. Encryption of data in the smartphone of the participant (in-app coding) with a 16-digit encryption key+a personal temporary code for access to data,

2. Transmission of the encrypted data to a secure server of a company based in France (Ivory Healthcare),

3. Physical transfer from the server to Nantes University Hospital secure medical server (USB stick or disk) at the end of the study period,

4. Once the data are uploaded on the intranet of the University Hospital, decryption in situ using specific software after entry of a short, automatically generated validation code by the principal investigator, and

5. Organisation of the obtained data in the form of a statistically exploitable Excel file.

\section{DISCUSSION}

Ecological naturalistic data on ASD patients are limited in the literature. The SMARTAUTISM application will be a unique source of such longitudinal observations.

The association of EMA with feedback in a carefully designed smartphone application might be valuable for three main reasons:

- The feedback provided by the application may be encouraging to users. Therefore, the filling rate should be higher and the epidemiological naturalistic data more accurate.

- The feedback might also be used by parents for contextualisation and adaptation of their educational behaviour. This aspect is crucial and will be evaluated in an ancillary study. The 'self-care' potential of
SMARTAUTISM, through personalised educational tips, is a major developmental project for us. We plan to develop a second version of the application.

- The feedback may also be shown to a doctor by the parents during regular consultations. Medical consultations can be conducted at wide intervals and cannot properly consider changes in symptoms from previous assessments or in everyday life hazards and the associated repercussions. The relationships of the patient and the family with their doctor may be profoundly changed by the use of this app. Since appointments with the patient's referring physician can be far apart, a major frustration is not being able to 'say everything'. Parties tend to speak only about the most recent events. For the doctors, this application can provide a perspective that more closely reflects reality and, above all, can reveal context that can aid in care and therapy. Therefore, using this application could generate a major breakthrough in the patient-doctor relationship. This issue will also be examined in an ancillary qualitative study. We plan to contact, with their permission, doctors who have used SMARTAUTISM with parents during consultations and ask about their impressions.

Our application is clearly a first step, but it is consistent with an ongoing shift towards the use of such technologies in psychiatry. Our goal is to use this application to regularly care for people with autism. Therefore, our research protocol is focused on feasibility (as demonstrated by the filling rates) and the quality of the data obtained. If, as we expect, the results are encouraging, we will proceed further.

\section{CONCLUSIONS}

The SMARTAUTISM project and research protocol are situated in the major changes in medical care that have been driven by the digital revolution. These changes affect our ability to obtain naturalistic epidemiological data reflecting real life and our capacity to provide medical care to patients and their families.

Acknowledgements The authors would like to acknowledge Julien Gachot and Matthieu Milliot, from IVORY HEALTHCARE for the development of the SMARTAUTISM App.

Contributors $\mathrm{OB}, \mathrm{DB}, \mathrm{PD}$ and $\mathrm{AD}$ were involved in the conception and design of the study. $O B$ drafted the article, $D B, P D$ and $A D$ revised it critically for important intellectual content. The figures were prepared by $A D$ and $O B . O B$, $\mathrm{DB}, \mathrm{AD}$ and $\mathrm{PD}$ were involved in the conception of the smartphone application. All authors gave final approval of the version to be published.

Funding Institut de Recherche en Epidémiologie et Santé Publique. Caisse Nationale de Solidarité pour l'Autonomie. Centre Ressource Autisme des Pays de la Loire.

Competing interests None declared.

Ethics approval Comité de Protection des Personnes en Recherche Biomédicales des Rennes.

Provenance and peer review Not commissioned; externally peer reviewed.

Open Access This is an Open Access article distributed in accordance with the Creative Commons Attribution Non Commercial (CC BY-NC 4.0) license, 
which permits others to distribute, remix, adapt, build upon this work noncommercially, and license their derivative works on different terms, provided the original work is properly cited and the use is non-commercial. See: http:// creativecommons.org/licenses/by-nc/4.0/

\section{REFERENCES}

1. Luton D. Medical diagnosis apps-study findings. This is social life. https://simplysociology.wordpress.com/2015/05/03/ medical-diagnosis-apps-study-findings/ (accessed 3 Dec 2015).

2. Brian RM, Ben-Zeev D. Mobile health (mHealth) for mental health in Asia: objectives, strategies, and limitations. Asian J Psychiatr 2014;10:96-100.

3. Shiffman RN. Informatics and general pediatrics. Curr Opin Pediatr 1994;6:538-43.

4. Csikszentmihalyi M, Larson R. Validity and reliability of the experience-sampling method. J Nerv Ment Dis 1987;175:526-36.

5. Shiffman S, Stone AA, Hufford MR. Ecological momentary assessment. Annu Rev Clin Psychol 2008:4:1-32.

6. Serre F, Fatseas M, Swendsen J, et al. Ecological momentary assessment in the investigation of craving and substance use in daily life: a systematic review. Drug Alcohol Depend 2015;148C:1-20.

7. Kikuchi H, Yoshiuchi K, Inada S, et al. Development of an ecological momentary assessment scale for appetite. Biopsychosoc Med 2015;9:2.

8. Walz LC, Nauta MH, Aan Het Rot M. Experience sampling and ecological momentary assessment for studying the daily lives of patients with anxiety disorders: a systematic review. J Anxiety Disord 2014;28:925-37.

9. Thompson WK, Gershon A, O'Hara R, et al. The prediction of study-emergent suicidal ideation in bipolar disorder: a pilot study using ecological momentary assessment data. Bipolar Disord 2014;16:669-77.

10. Granholm E, Ben-Zeev D, Fulford D, et al. Ecological momentary assessment of social functioning in schizophrenia: impact of performance appraisals and affect on social interactions. Schizophr Res 2013;145:120-4.

11. Husky M, Olié E, Guillaume S, et al. Feasibility and validity of ecological momentary assessment in the investigation of suicide risk. Psychiatry Res 2014;220:564-70.

12. Torous J, Friedman R, Keshavan M. Smartphone ownership and interest in mobile applications to monitor symptoms of mental health conditions. JMIR Mhealth Uhealth 2014;2:e2.

13. Ben-Zeev D, Kaiser SM, Brenner CJ, et al. Development and usability testing of FOCUS: a smartphone system for self-management of schizophrenia. Psychiatr Rehabil J 2013;36:289-96.
14. Gajecki M, Berman AH, Sinadinovic K, et al. Mobile phone brief intervention applications for risky alcohol use among university students: a randomized controlled study. Addict Sci Clin Pract 2014;9:11.

15. Grünerbl A, Muaremi A, Osmani V, et al. Smart-phone based recognition of states and state changes in bipolar disorder patients. IEEE J Biomed Health Inform 2014:19:140-8.

16. Faurholt-Jepsen M, Vinberg M, Christensen EM, et al. Daily electronic self-monitoring of subjective and objective symptoms in bipolar disorder-the MONARCA trial protocol (MONitoring, treAtment and pRediCtion of bipolAr disorder episodes): a randomised controlled single-blind trial. BMJ Open 2013;3: e003353.

17. Dag öö J, Asplund RP, et al. Cognitive behavior therapy versus interpersonal psychotherapy for social anxiety disorder delivered via smartphone and computer: a randomized controlled trial. J Anxiety Disord 2014;28:410-17.

18. Ben-Zeev D, Brenner CJ, Begale M, et al. Feasibility, acceptability, and preliminary efficacy of a smartphone intervention for schizophrenia. Schizophr Bull 2014;40:1244-53.

19. Begovac I, Divcić B, Begovac B, et al. The loss of criteria for mental retardation and regression of symptoms of childhood autism during a five-year follow-up-a case report. Acta Clin Croat 2009;48:445-50

20. Gor RA, Fuhrer J, Schober JM. A retrospective observational study of enuresis, daytime voiding symptoms, and response to medical therapy in children with attention deficit hyperactivity disorder and autism spectrum disorder. J Pediatr Urol 2012;8:314-17.

21. Baghdadli A, Pry R, Michelon C, et al. Impact of autism in adolescents on parental quality of life. Qual Life Res 2014;23:1859-68

22. Farnik M, Brozek G, Pierzchała W, et al. Development, evaluation and validation of a new instrument for measurement quality of life in the parents of children with chronic disease. Health Qual Life Outcomes 2010;8:151.

23. Hayes SA, Watson SL. The impact of parenting stress: a meta-analysis of studies comparing the experience of parenting stress in parents of children with and without autism spectrum disorder. J Autism Dev Disord 2013:43:629-42.

24. Davis NO, Carter AS. Parenting stress in mothers and fathers of toddlers with autism spectrum disorders: associations with child characteristics. J Autism Dev Disord 2008;38:1278-91.

25. Henry C, M'Bailara K, Mathieu F, et al. Construction and validation of a dimensional scale exploring mood disorders: MAThyS (Multidimensional Assessment of Thymic States). BMC Psychiatry 2008;8:82.

26. Schopler E, Reichler RJ, DeVellis RF, et al. Toward objective classification of childhood autism: Childhood Autism Rating Scale (CARS). J Autism Dev Disord 1980;10:91-103. 